\title{
Note sur les formules de saut de Guillemin-Kalkman
}

\author{
A note on the formulas of Guillemin-Kalkman \\ Paul-Émile Paradan \\ UMR 5582, Institut Fourier, B.P. 74, 38402, Saint-Martin-d'Hères cedex, France
}

\begin{abstract}
The purpose of this note is to recover the formulas of Guillemin-Kalkman [2] from jump formulas in equivariant cohomology.
\end{abstract}

\section{Résumé}

Le but de cette note est de donner une formule de saut en cohomologie équivariante qui entraîne les formules de saut de Guillemin-Kalkman [2].

\section{Abridged English version}

Let $(M, \omega)$ be a symplectic manifold equipped with a Hamiltonian action of a torus $T$, with Lie algebra $\mathfrak{t}$. We denote by $\Phi: M \rightarrow \mathfrak{t}^{*}$ the moment map of this action. Let us assume that $\Phi$ is proper, and that the $T$-action on $M$ is effective. For every regular value $\xi$ of $\Phi$, we consider the reduction $\mathcal{M}_{\xi}:=\Phi^{-1}(\xi) / T$ which is a compact symplectic orbifold. Let $\mathcal{H}_{T}^{*}(M)$ be the $T$-equivariant cohomology of $M$. Associated to the data $(M, T, \Phi)$ we have, for every regular value $\xi$ of $\Phi$, the $\operatorname{Kirwan}$ morphism $\operatorname{Kir}_{M, \xi}: \mathcal{H}_{T}^{*}(M) \rightarrow$ $\mathcal{H}^{*}\left(\mathcal{M}_{\xi}\right)$ [4]. We are interested here in $\mathrm{I}(M, \eta, \xi):=\frac{1}{\left|S_{\xi}\right|} \int_{\mathcal{M}_{\xi}} \operatorname{Kir}_{M, \xi}(\eta), \eta \in \mathcal{H}_{T}^{*}(M)$, where $\left|S_{\xi}\right|$ is the cardinal of the generic stabilizer of $T$ on $\Phi^{-1}(\xi)$.

We introduce for every $\xi \in \mathfrak{t}^{*}$ an equivariant cohomology class with compact support $\mathrm{P}_{\xi} \in \mathcal{H}_{T, c}^{-\infty}(M)$, and show that $\mathrm{I}(M, \eta, \xi)$ is computed by means of $\mathrm{P}_{\xi}$. If $\xi$ is a regular value of $\Phi$, the integral $\int_{M} \mathrm{P}_{\xi} \eta$ is a generalized function on $\mathfrak{t}$ supported at 0 . The quantity $\mathrm{I}(M, \eta, \xi)$ is equal to the value of $\int_{M} \mathrm{P}_{\xi} \eta$ against $(2 i \pi)^{-\operatorname{dim} T} \frac{d X}{\operatorname{vol}(T, d X)}$. The study of the map $\xi \mapsto \mathrm{P}_{\xi}$ gives a new way to recover two properties of the map $\xi \rightarrow \mathrm{I}(M, \eta, \xi)$. First we show that $\xi \mapsto \mathrm{P}_{\xi}$ is locally constant on the open subset of regular values

Email address: Paul-Emile.Paradan@ujf-grenoble.fr (Paul-Émile Paradan). 
of $\Phi$, so $\xi \rightarrow \mathrm{I}(M, \eta, \xi)$ is also locally constant. After we obtain a formula for $\mathrm{P}_{\xi^{+}}-\mathrm{P}_{\xi^{-}}$when $\xi^{ \pm}$belong respectively to two connected components of regular values of $\Phi$ separated by an hyperplane of $t^{*}$. By this way we recover the formulas of Guillemin-Kalkman [2]. Let us write down these formulas.

Let $\Delta$ be an hyperplane of $\mathfrak{t}^{*}$, equipped with an orientation $o$, and which separates two connected components of regular values of $\Phi$. Let $T_{\Delta} \subset T$ be the subtorus of dimension 1 , with Lie algebra $\mathfrak{t}_{\Delta}:=$ $\left\{X \in \mathfrak{t} \mid\left\langle\xi-\xi^{\prime}, X\right\rangle=0, \forall \xi, \xi^{\prime} \in \Delta\right\}$. We make the choice of a decomposition $T=T_{\Delta} \times T / T_{\Delta}$, where $T / T_{\Delta}$ denotes a subtorus de $T$. Let $M^{T_{\Delta}}$ be the submanifold of points fixed by $T_{\Delta}$, and let $M_{\Delta}$ be the open subset of $M^{T_{\Delta}} \cap \Phi^{-1}(\Delta)$ on which $T / T_{\Delta}$ acts locally freely. The symplectic manifold $M_{\Delta}$ carries a Hamiltonian action of $T / T_{\Delta}$ with moment map $\Phi_{\Delta}: M_{\Delta} \rightarrow \Delta$ equal to the restriction of $\Phi$ on $M_{\Delta}$.

Let $\xi \in \Delta$ be a regular value of $\Phi_{\Delta}$ and let $\mathrm{P}_{\xi}^{\Delta} \in \mathcal{H}_{T / T_{\Delta}, c}^{-\infty}\left(M_{\Delta}\right)$ be the associated cohomology class. Let $\xi^{ \pm} \in \mathfrak{t}^{*}$ be two regular values of $\Phi$ belonging respectively to two connected components of regular values of $\Phi$ separated by $\Delta$, with the condition that the line $\left(\xi^{+}, \xi^{-}\right)$intersects $\Delta$ at $\xi$ and $\xi^{-}-\xi^{+}$is compatible with the orientation $o$. We prove in this note that

$$
\mathrm{P}_{\xi^{+}}-\mathrm{P}_{\xi^{-}}=\left(i_{\Delta}\right)_{*}\left(\mathrm{P}_{\xi}^{\Delta} \delta_{\Delta}^{o}\right) \quad \text { in } \quad \mathcal{H}_{T, c}^{-\infty}(M)
$$

Here $\left(i_{\Delta}\right)_{*}: \mathcal{H}_{T, c}^{-\infty}\left(M_{\Delta}\right) \rightarrow \mathcal{H}_{T, c}^{-\infty}(M)$ is the direct image map, and $\delta_{\Delta}^{o} \in \mathcal{C}^{-\infty}\left(\mathfrak{t}_{\Delta}, \mathcal{H}^{*}\left(M_{\Delta}\right)^{\text {bas }}\right)$ where $\mathcal{H}^{*}\left(M_{\Delta}\right)^{\text {bas }}$ is the sub-algebra of $\mathcal{H}^{*}\left(M_{\Delta}\right)$ formed by the $T$-basic elements. With $\delta_{\Delta}^{o}$ one defines a residue map $\operatorname{Res}_{\Delta}^{o}: \mathcal{H}_{T}^{*}(M) \rightarrow \mathcal{H}_{T / T_{\Delta}}^{*}\left(M_{\Delta}\right)$, and from (1) we get the formulas of Guillemin-Kalkman

$$
\mathrm{I}\left(M, \eta, \xi^{+}\right)-\mathrm{I}\left(M, \eta, \xi^{-}\right)=\mathrm{I}\left(M_{\Delta}, \operatorname{Res}_{\Delta}^{o}(\eta), \xi\right),
$$

for every $\eta \in \mathcal{H}_{T}^{*}(M)$. The class $\delta_{\Delta}^{o}$ and the map $\operatorname{Res}_{\Delta}^{o}$ are defined in Section 2.

\section{Introduction}

Soit $(M, \omega)$ une variété symplectique munie de l'action hamiltonienne d'un tore $T$ d'algèbre de Lie $\mathfrak{t}$. L'application moment $\Phi: M \rightarrow \mathfrak{t}^{*}$ satisfait les relations $d\langle\Phi, X\rangle+\omega\left(X_{M},-\right)=0, \forall X \in \mathfrak{t}$. On suppose dans cette note que $\Phi$ est propre, et que l'action de $T$ sur $M$ est effective. Pour toute valeur régulière $\xi$ de $\Phi$, on considère la réduction symplectique $\mathcal{M}_{\xi}:=\Phi^{-1}(\xi) / T$ qui est une $V$-variété différentiable compacte. Soit $\mathcal{H}_{T}^{*}(M)$ la cohomologie $T$-équivariante de $M$. Sur la variété hamiltonienne $(M, T, \Phi)$ nous

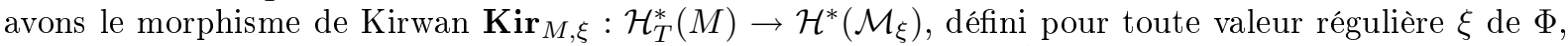
comme le composé du morphisme de restriction $\mathcal{H}_{T}^{*}(M) \rightarrow \mathcal{H}_{T}^{*}\left(\Phi^{-1}(\xi)\right)$ avec l'isomorphisme de ChernWeil $\mathcal{H}_{T}^{*}\left(\Phi^{-1}(\xi)\right) \stackrel{\sim}{\longrightarrow} \mathcal{H}^{*}\left(\mathcal{M}_{\xi}\right)$. Nous nous intéressons ici à $\mathrm{I}(M, \eta, \xi):=\frac{1}{\left|S_{\xi}\right|} \int_{\mathcal{M}_{\xi}} \mathbf{K i r}_{M, \xi}(\eta), \eta \in \mathcal{H}_{T}^{*}(M)$, où $\left|S_{\xi}\right|$ est le cardinal du stabilisateur générique de $T$ sur $\Phi^{-1}(\xi)$.

On introduit à la section 2 une classe $\mathrm{P}_{\xi} \in \mathcal{H}_{T, c}^{-\infty}(M)$ pour tout $\xi \in \mathfrak{t}^{*}$, et on montre que $\mathrm{I}(M, \eta, \xi)$ se calcule au moyen de $\mathrm{P}_{\xi}$. L'étude de l'application $\xi \mapsto \mathrm{P}_{\xi}$ entreprise à la section 3 permet de redémontrer deux propriétés de $\xi \mapsto \mathrm{I}(M, \eta, \xi)$. On montre tout d'abord que $\xi \mapsto \mathrm{P}_{\xi}$ est localement constante sur l'ouvert des valeurs régulières de $\Phi$. On obtient ensuite des formules de saut pour les classes $\mathrm{P}_{\xi}$ qui induisent celles pour $\mathrm{I}(M, \eta, \xi)$ obtenues par Guillemin-Kalkman [2]. Ces formules s'énoncent ainsi.

Soit $\Delta$ un hyperplan de $\mathfrak{t}^{*}$, muni d'une orientation $o$, séparant deux composantes connexes de l'ouvert des points réguliers de $\Phi$. Soit $T_{\Delta} \subset T$ le sous-tore de dimension 1 , d'algèbre de Lie $\mathfrak{t}_{\Delta}:=$ $\left\{X \in \mathfrak{t} \mid\left\langle\xi-\xi^{\prime}, X\right\rangle=0, \forall \xi, \xi^{\prime} \in \Delta\right\}$. Nous faisons le choix d'une décomposition $T=T_{\Delta} \times T / T_{\Delta}$, où $T / T_{\Delta}$ désigne un sous-tore de $T$. Considérons la sous-variété $M^{T_{\Delta}}$ des points fixés par $T_{\Delta}$, et l'ouvert $T$-invariant $M_{\Delta}$ de $\Phi^{-1}(\Delta) \cap M^{T_{\Delta}}$ sur lequel $T / T_{\Delta}$ agit localement librement. La variété symplectique $M_{\Delta}$ est munie de l'action hamiltonienne de $T / T_{\Delta}$ et a pour application moment la restriction de $\Phi$ à $M_{\Delta}$, que l'on note $\Phi_{\Delta}: M_{\Delta} \rightarrow \Delta$. 
Considérons une valeur régulière $\xi \in \Delta$ de $\Phi_{\Delta}$ et la classe de cohomologie équivariante associée $\mathrm{P}_{\xi}^{\Delta} \in$ $\mathcal{H}_{T / T_{\Delta}, c}^{-\infty}\left(M_{\Delta}\right)$. Soient $\xi^{ \pm} \in \mathfrak{t}^{*}$ deux valeurs régulières de $\Phi$ appartenant à deux composantes connexes de valeurs régulières de $\Phi$ séparées par $\Delta$ : on suppose que le segment $\left(\xi^{+}, \xi^{-}\right)$intersecte $\Delta$ en $\xi$ et que $\xi^{-}-\xi^{+}$est compatible avec l'orientation $o$. On montre à la proposition 1 que

$$
\mathrm{P}_{\xi^{+}}-\mathrm{P}_{\xi^{-}}=\left(i_{\Delta}\right)_{*}\left(\mathrm{P}_{\xi}^{\Delta} \delta_{\Delta}^{o}\right) \quad \text { dans } \quad \mathcal{H}_{T, c}^{-\infty}(M)
$$

Ici $\left(i_{\Delta}\right)_{*}: \mathcal{H}_{T, c}^{-\infty}\left(M_{\Delta}\right) \rightarrow \mathcal{H}_{T, c}^{-\infty}(M)$ est le morphisme image directe associé à l'inclusion $i_{\Delta}: M_{\Delta} \hookrightarrow M$, et $\delta_{\Delta}^{o}$ est une fonction généralisée sur $\mathfrak{t}_{\Delta}$ à valeurs dans la sous-algèbre $\mathcal{H}^{*}\left(M_{\Delta}\right)^{\text {bas }}$ de $\mathcal{H}^{*}\left(M_{\Delta}\right)$ formée des éléments $T$-basiques. On définit au moyen de $\delta_{\Delta}^{o}$ on définit une application résidu $\operatorname{Res}_{\Delta}^{o}: \mathcal{H}_{T}^{*}(M) \rightarrow$ $\mathcal{H}_{T / T_{\Delta}}^{*}\left(M_{\Delta}\right)$, et on déduit de (3) les formules de sauts de Guillemin-Kalkman

$$
\mathrm{I}\left(M, \eta, \xi^{+}\right)-\mathrm{I}\left(M, \eta, \xi^{-}\right)=\mathrm{I}\left(M_{\Delta}, \operatorname{Res}_{\Delta}^{o}(\eta), \xi\right),
$$

pour tout $\eta \in \mathcal{H}_{T}^{*}(M)$. La fonction $\delta_{\Delta}^{o}$ et l'application $\operatorname{Res}_{\Delta}^{o}$ sont définis à la prochaine section.

\section{Cohomologie équivariante : localisation et définition de l'application $\operatorname{Res}_{\Delta}^{o}$}

Rappelons le modèle de Cartan de la cohomologie équivariante à coefficients polynomiaux, et l'extension aux coefficients généralisés définie par Kumar-Vergne [5]. Nous donnons ensuite un bref aperçu de la méthode de localisation développée dans [6,7], et donnons la définition de l'application $\operatorname{Res}_{\Delta}^{o}$.

Soit $K$ un groupe de Lie compact connexe, d'algèbre de Lie $\mathfrak{k}$, agissant de manière $\mathcal{C}^{\infty}$ sur une variété différentiable $M$. On note $\mathcal{A}(M)$ l'algèbre sur $\mathbb{C}$ des formes différentielles $\mathcal{C}^{\infty}$ et $d$ la dérivation de de Rham. On note $c(V): \mathcal{A}(M) \rightarrow \mathcal{A}(M)$ la contraction par un champ de vecteurs $V$. L'action de $K$ sur $M$ détermine un morphisme $X \rightarrow X_{M}$ de $\mathfrak{k}$ dans l'algèbre des champs de vecteurs de $M$.

On considère l'espace des applications $K$-équivariantes $\mathfrak{k} \rightarrow \mathcal{A}(M), X \mapsto \eta(X)$, muni de la dérivation $(D \eta)(X):=\left(d-c\left(X_{M}\right)\right)(\eta(X)), X \in \mathfrak{k}$. Comme $D^{2}=0$, on peut considérer l'espace de cohomologie $\operatorname{Ker} D / \operatorname{Im} D$. Le modèle de Cartan [1,3] considère des applications polynomiales, et l'espace de cohomologie associé est noté $\mathcal{H}_{K}^{*}(M)$. Kumar et Vergne [5] ont étudié les espaces de cohomologie $\mathcal{H}_{K}^{ \pm \infty}(M)$ obtenus en considérant des applications $X \mapsto \eta(X)$ qui sont $\mathcal{C}^{ \pm \infty}$. Rappelons la construction de $\mathcal{H}_{K}^{-\infty}(M)$.

Soit $\mathcal{C}^{-\infty}(\mathfrak{k}, \mathcal{A}(M))$ l'espace des fonctions généralisées sur $\mathfrak{k}$ à valeurs dans $\mathcal{A}(M)$. C'est, par définition, l'espace des applications $\mathbb{C}$-linéaires continues de l'espace des densités $\mathcal{C}^{\infty}$ à support compact de $\mathfrak{k}$ dans $\mathcal{A}(M)$. L'image de la densité $\phi(X) d X$ par $\eta \in \mathcal{C}^{-\infty}(\mathfrak{k}, \mathcal{A}(M))$ est une forme différentielle sur $M$ notée $\int_{\mathfrak{k}} \eta(X) \phi(X) d X$. La différentielle $D$ définie sur $\mathcal{C}^{\infty}(\mathfrak{k}, \mathcal{A}(M))$ se prolonge à $\mathcal{C}^{-\infty}(\mathfrak{k}, \mathcal{A}(M))$, et on montre que $D^{2}=0$ sur le sous-espace $\mathcal{C}^{-\infty}(\mathfrak{k}, \mathcal{A}(M))^{K}$ des éléments $K$-invariants [5]. L'espace de cohomologie associé est la cohomologie $K$-équivariante à coefficients généralisés de $M$, que l'on note $\mathcal{H}_{K}^{-\infty}(M)$. Si nous considérons des formes équivariantes à valeurs dans l'espace $\mathcal{A}_{c}(M)$ des formes différentielles à support compact on obtient l'espace de cohomologie $\mathcal{H}_{K, c}^{-\infty}(M)$. Dans cette note, on note $\operatorname{vol}(K, d X)$ le volume du groupe $K$ pour la mesure de Haar compatible avec $d X$.

Procédé de localisation. On suppose désormais que $M$ est munie d'une 1 -forme $\lambda K$-invariante. On note $\Phi_{\lambda}: M \rightarrow \mathfrak{k}^{*}$ l'application équivariante définie par $\left\langle\Phi_{\lambda}(m), X\right\rangle=\lambda\left(X_{M}\right)_{m}$ : on a donc $D \lambda(X)=$ $d \lambda-\left\langle\Phi_{\lambda}, X\right\rangle$. Le procédé de localisation développé en [6,7] repose sur l'existence d'un inverse $[D \lambda]^{-1} \mathrm{de}$ la forme $K$-équivariante $D \lambda$ : c'est une forme $K$-équivariante fermée à coefficients généralisés définie sur l'ouvert $M-\Phi_{\lambda}^{-1}(0)$. Un ouvert $K$-invariant $\mathcal{U}$ est dit adapté à $\lambda$ si $(\partial \mathcal{U}) \cap \Phi_{\lambda}^{-1}(0)=\emptyset$. Dans [7], on associe à tout ouvert $\mathcal{U}$ adapté à $\lambda$, la forme $K$-équivariante fermée à coefficients généralisés

$$
\mathrm{P}_{\lambda}^{\mathcal{U}}=\chi^{\mathcal{U}}+d \chi^{\mathcal{U}}[D \lambda]^{-1} \lambda
$$


Ici $\chi^{\mathcal{U}} \in \mathcal{C}^{\infty}(M)$ désigne une fonction $K$-invariante supportée par $\mathcal{U}$ qui est égale à 1 au voisinage de $\mathcal{U} \cap \Phi_{\lambda}^{-1}(0)$. La classe définie par $\mathrm{P}_{\lambda}^{\mathcal{U}}$ dans $\mathcal{H}_{K}^{-\infty}(M)$ ne dépend pas du choix de $\chi^{\mathcal{U}}$. Si $\mathcal{U} \cap \Phi_{\lambda}^{-1}(0)$ est compact, on considère une fonction $\chi^{\mathcal{U}}$ à support compact : ainsi $\mathrm{P}_{\lambda}^{\mathcal{U}}$ définit une classe dans $\mathcal{H}_{K, c}^{-\infty}(M)$. La correspondance $\mathcal{U} \rightarrow \mathrm{P}_{\lambda}^{\mathcal{U}}$ est additive dans le sens suivant. Si $\mathcal{U}$ contient deux ouverts $\mathcal{U}^{1}, \mathcal{U}^{2} K$-invariants tels que $\overline{\mathcal{U}^{1}} \cap \overline{\mathcal{U}^{2}} \cap \Phi_{\lambda}^{-1}(0)=\emptyset$ et $\left(\mathcal{U}^{1} \cup \mathcal{U}^{2}\right) \cap \Phi_{\lambda}^{-1}(0)=\mathcal{U} \cap \Phi_{\lambda}^{-1}(0)$, alors les $\mathcal{U}^{i}$ sont adaptés à $\lambda$ et $\mathrm{P}_{\lambda}^{\mathcal{U}}=\mathrm{P}_{\lambda}^{\mathcal{U}^{1}}+\mathrm{P}_{\lambda}^{\mathcal{U}^{2}}$ dans $\mathcal{H}_{T}^{-\infty}(M)$. Pour l'étude des classes de cohomologies définies par les $\mathrm{P}_{\lambda}^{\mathcal{U}}$ on a le

Lemme 2.1 ([7]) Soient $\lambda_{0}, \lambda_{1}$ deux 1-formes $K$-invariantes sur $M$, et $\mathcal{U}$ un ouvert K-invariant relativement compact de $M$. S'il existe une application continue $f: M \rightarrow \mathfrak{k}$ telle que les fonctions $\left\langle\Phi_{\lambda_{0}}, f\right\rangle$ et

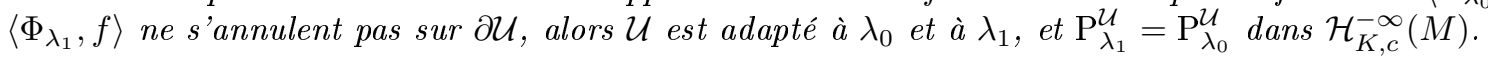

Défnitions de $\delta_{\Delta}^{o}$ et de l'application $\operatorname{Res}_{\Delta}^{o}: \mathcal{H}_{T}^{*}(M) \rightarrow \mathcal{H}_{T / T_{\Delta}}^{*}\left(M_{\Delta}\right)$. On revient au cadre de l'introduction. La décomposition $T=T_{\Delta} \times T / T_{\Delta}$, l'action triviale de $T_{\Delta}$ sur $M_{\Delta}$ et l'action localement libre de $T / T_{\Delta}$ sur $M_{\Delta}$ induisent les isomorphismes $j_{\Delta}: \mathcal{H}_{T}^{*}\left(M_{\Delta}\right) \stackrel{\sim}{\longrightarrow} \mathcal{S}^{*}\left(\mathfrak{t}_{\Delta}\right) \otimes \mathcal{H}_{T / T_{\Delta}}^{*}\left(M_{\Delta}\right)$ et $\mathbf{c v}_{\Delta}: \mathcal{H}_{T / T_{\Delta}}^{*}\left(M_{\Delta}\right) \stackrel{\sim}{\longrightarrow}$ $\mathcal{H}^{*}\left(M_{\Delta}\right)^{\text {bas }}$. Ici $\mathcal{S}^{*}\left(\mathfrak{t}_{\Delta}\right)$ désigne l'algèbre des applications polynomiales sur $\mathfrak{t}_{\Delta}$ et $\mathcal{H}^{*}\left(M_{\Delta}\right)^{\text {bas }}$ la sousalgèbre de $\mathcal{H}^{*}\left(M_{\Delta}\right)$ formée des éléments $T$-basiques. On note $\mathbf{K i r}^{\Delta}: \mathcal{H}_{T}^{*}(M) \rightarrow \mathcal{S}^{*}\left(\mathfrak{t}_{\Delta}\right) \otimes \mathcal{H}^{*}\left(M_{\Delta}\right)^{\text {bas }}$ le composé du morphisme de restriction $\mathcal{H}_{T}^{*}(M) \rightarrow \mathcal{H}_{T}^{*}\left(M_{\Delta}\right)$ avec l'isomorphisme $\mathbf{c v}_{\Delta} \circ j_{\Delta}$.

Soient $N_{\Delta}$ le fibré normal $T$-équivariant de $M_{\Delta}$ dans $M$, et $\operatorname{Eul}\left(N_{\Delta}\right) \in \mathcal{H}_{T}^{*}\left(M_{\Delta}\right)$ la classe d'Euler $T$-équivariante de $N_{\Delta}$. A travers l'isomorphisme $\mathbf{c v}_{\Delta} \circ j_{\Delta}$ on peut considérer $\operatorname{Eul}\left(N_{\Delta}\right)$ comme un élément de $\mathcal{S}^{*}\left(\mathfrak{t}_{\Delta}\right) \otimes \mathcal{H}^{*}\left(M_{\Delta}\right)^{\text {bas }}$. Suivant [6], on définit des inverses $\operatorname{Eul}_{ \pm}^{-1}\left(N_{\Delta}\right) \in \mathcal{C}^{-\infty}\left(\mathfrak{t}_{\Delta}, \mathcal{H}^{*}\left(M_{\Delta}\right)^{\text {bas }}\right)$ en posant $\operatorname{Eul}_{ \pm}^{-1}\left(N_{\Delta}\right)(X)=\lim _{s \rightarrow+\infty} \frac{1}{\operatorname{Eul}\left(N_{\Delta}\right)(X \pm i s \beta)}$, où $\beta \in \mathfrak{t}_{\Delta}-\{0\}$ est compatible avec l'orientation $o$ de $\Delta$. Comme le polynôme $\operatorname{Eul}\left(N_{\Delta}\right)$ est inversible de manière $\mathcal{C}^{\infty}$ sur $\mathfrak{t}_{\Delta}-\{0\}$, la différence

$$
\delta_{\Delta}^{o}:=\operatorname{Eul}_{-}^{-1}\left(N_{\Delta}\right)-\operatorname{Eul}_{+}^{-1}\left(N_{\Delta}\right)
$$

est une fonction généralisée sur $\mathfrak{t}_{\Delta}$ qui est supportée en 0 (à valeurs dans $\mathcal{H}^{*}\left(M_{\Delta}\right)^{\text {bas }}$ ). Soit $r_{\Delta}^{o}: \mathcal{S}^{*}\left(\mathfrak{t}_{\Delta}\right) \otimes$ $\mathcal{H}^{*}\left(M_{\Delta}\right)^{\text {bas }} \rightarrow \mathcal{H}^{*}\left(M_{\Delta}\right)^{\text {bas }}$ définie par $r_{\Delta}^{o}(P)=\frac{1}{2 i \pi} \int_{\mathfrak{t}_{\Delta}} \delta_{\Delta}^{o}(X) P(X) \frac{d X}{\operatorname{vol}\left(T_{\Delta}, d X\right)}$. L'application $\operatorname{Res}_{\Delta}^{o}:$ $\mathcal{H}_{T}^{*}(M) \rightarrow \mathcal{H}_{T / T_{\Delta}}^{*}\left(M_{\Delta}\right)$ est définie par le diagramme commutatif suivant

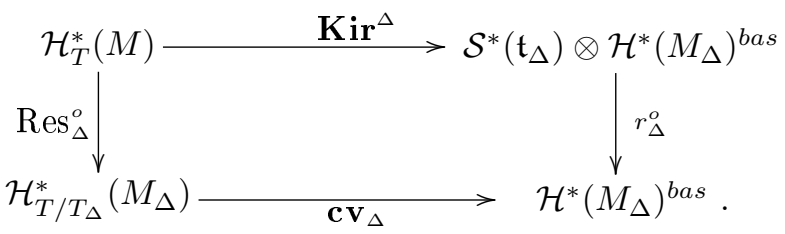

\section{Quelques propriétés de l'application $\xi \mapsto \mathrm{I}(M, \eta, \xi)$}

Nous revenons au contexte d'une action hamiltonienne d'un tore $T$ sur une variété symplectique $(M, \omega)$. On garde les mêmes notations et hypothèses que dans l'introduction. On fixe un produit scalaire sur $\mathfrak{t}^{*}$ qui induit une identification $\mathfrak{t}^{*} \simeq \mathfrak{t}$. On fixe sur $M$ une structure riemannienne $T$-invariante, notée $(-,-)_{M}$.

\subsection{Caractérisation de $\mathrm{I}(M, \eta, \xi)$}

Soit $\mathcal{H}$ le champ de vecteurs hamiltonien de la fonction $\frac{1}{2}\|\Phi\|^{2}$. Alors, pour tout $\xi \in \mathfrak{t}^{*}$, le champ de vecteurs hamiltonien de la fonction $\frac{1}{2}\|\Phi-\xi\|^{2}$ est $\mathcal{H}-\xi_{M}$. Considérons la 1-forme $T$-invariante $\lambda_{\xi}=$ 
$\left(\mathcal{H}-\xi_{M},-\right)_{M}$ et l'application $\Phi_{\lambda_{\xi}}: M \rightarrow \mathfrak{t}^{*}$ associée (voir section 2). Ici $\Phi_{\lambda_{\xi}}^{-1}(0)$ coïncide avec le sousensemble $\operatorname{Cr}\left(\|\Phi-\xi\|^{2}\right) \subset M$ des points critiques de la fonction $\|\Phi-\xi\|^{2}$, et d'autre part $\left.m \in \operatorname{Cr}\left(\|\Phi-\xi\|^{2}\right)\right)$ si et seulement si $(\Phi(m)-\xi)_{M}$ s'annule en $\mathrm{m}$. Considérons la classe de cohomologie

$$
\mathrm{P}_{\xi} \in \mathcal{H}_{T, c}^{-\infty}(M)
$$

définie par $\mathrm{P}_{\lambda_{\xi}}^{\mathcal{U}}$, où $\mathcal{U}$ est un voisinage ouvert $T$-invariant relativement compact de $\Phi^{-1}(\xi)$ tel que $\overline{\mathcal{U}} \cap$ $\operatorname{Cr}\left(\|\Phi-\xi\|^{2}\right)=\Phi^{-1}(\xi)$.

On rappelle maintenant le calcul de $\mathrm{P}_{\xi}$ lorsque $\xi$ est une valeur régulière de $\Phi[6,7]$. Soit $\Omega_{\xi} \in \mathcal{H}^{2}\left(\mathcal{M}_{\xi}\right) \otimes \mathfrak{t}$ la courbure du $T$-fibré principal $\Phi^{-1}(\xi) \rightarrow \mathcal{M}_{\xi}$. Pour toute fonction $\psi \in \mathcal{C}^{\infty}(\mathfrak{t})$, on désigne par $\psi\left(\Omega_{\xi}\right) \in$ $\mathcal{H}^{*}\left(\mathcal{M}_{\xi}\right)$ la valeur de l'opérateur différentiel $e^{\Omega_{\xi}\left(\left.\frac{\partial}{\partial X}\right|_{0}\right)}$ contre la fonction $\psi$. D'après [7][Prop. 3.11], la fonction généralisée $\int_{M} \mathrm{P}_{\xi} \eta$ est supportée en 0 pour tout $\eta \in \mathcal{H}_{T}^{*}(M)$ et

$$
\int_{M \times \mathfrak{t}} \mathrm{P}_{\xi}(X) \eta(X) \psi(X) d X=\frac{(2 i \pi)^{d i m T} \operatorname{vol}(T, d X)}{\left|S_{\xi}\right|} \int_{\mathcal{M}_{\xi}} \operatorname{Kir}_{M, \xi}(\eta) \psi\left(\Omega_{\xi}\right) .
$$

Pour une fonction généralisée $g \in \mathcal{C}^{-\infty}(\mathfrak{t})$ supportée en 0 , on définit sa multiplicité par rapport à la masse de Dirac en 0 comme la quantité $\int_{\mathfrak{t}} g(X) \frac{d X}{\operatorname{vol}(T, d X)} \in \mathbb{C}$. Sachant que $\psi\left(\Omega_{\xi}\right)=1$ si $\psi=1$, on obtient

Lemme 3.1 Pour toute valeur régulière $\xi$ de $\Phi$, l'intégrale $\mathrm{I}(M, \eta, \xi)$ est égale à la multiplicité par rapport à la masse de Dirac en 0 de la fonction généralisée $(2 i \pi)^{-\operatorname{dim} T} \int_{M} \mathrm{P}_{\xi} \eta$.

On ramène ainsi l'étude de l'application $\xi \mapsto \mathrm{I}(M, \eta, \xi)$ à celle de l'application $\xi \mapsto \mathrm{P}_{\xi}$.

\subsection{Deux propriétés de l'application $\xi \mapsto \mathrm{P}_{\xi}$.}

Fixons $\xi \in \mathfrak{t}^{*}$ et considérons l'application $f=\Phi-\xi$. Pour $\xi^{\prime} \in \mathfrak{t}^{*}$ on a $\left\langle\Phi_{\lambda_{\xi^{\prime}}}, f\right\rangle=\left(\mathcal{H}-\xi_{M}^{\prime}, \mathcal{H}-\xi_{M}\right)_{M}=$ $\left\|\mathcal{H}-\xi_{M}\right\|^{2}+\left(\left(\xi-\xi^{\prime}\right)_{M}, \mathcal{H}-\xi_{M}\right)_{M}$ ce qui donne $\left\langle\Phi_{\lambda_{\xi^{\prime}}}, f\right\rangle \geq\left\|\mathcal{H}-\xi_{M}\right\|\left(\left\|\mathcal{H}-\xi_{M}\right\|-\left\|\left(\xi-\xi^{\prime}\right)_{M}\right\|\right)(*)$. Soit $\mathcal{U}$ un voisinage ouvert, relativement compact et $T$-invariant de $\Phi^{-1}(\xi)$ tel que $\overline{\mathcal{U}} \cap \operatorname{Cr}\left(\|\Phi-\xi\|^{2}\right)=\Phi^{-1}(\xi)$ : alors $\mathrm{P}_{\xi}=\mathrm{P}_{\lambda_{\xi}}^{\mathcal{U}}$ dans $\mathcal{H}_{T}^{-\infty}(M)$. Sur $\partial \mathcal{U}$ (une partie compacte de $M$ ), le champ de vecteurs $\mathcal{H}-\xi_{M}$ ne s'annulant pas, on a des encadrements de la forme $\left\|\mathcal{H}-\xi_{M}\right\| \geq c_{1}>0$ et $\left\|a_{M}\right\| \leq c_{2}\|a\|, \forall a \in \mathfrak{t}$. Finalement, avec $(*)$ on obtient sur $\partial \mathcal{U}$ la minoration $\left\langle\Phi_{\lambda_{\xi^{\prime}}}, f\right\rangle \geq c_{1}\left(c_{1}-c_{2}\left\|\xi-\xi^{\prime}\right\|\right)$, et donc $\left\langle\Phi_{\lambda_{\xi^{\prime}}}, f\right\rangle \geq c_{3}>0$, pour $\xi^{\prime}$ suffisamment proche de $\xi$. Le lemme 2.1 montre alors que $\mathcal{U}$ est adapté à $\lambda_{\xi^{\prime}}$ et que

$$
\mathrm{P}_{\lambda_{\xi^{\prime}}}^{\mathcal{U}}=\mathrm{P}_{\lambda_{\xi}}^{\mathcal{U}}=\mathrm{P}_{\xi} \quad \text { dans } \quad \mathcal{H}_{T, c}^{-\infty}(M) \text { pour } \xi^{\prime} \text { suffisamment proche de } \xi \text {. }
$$

Considérons tout d'abord le cas où $\xi$ est une valeur régulière de $\Phi$. Alors $\overline{\mathcal{U}} \subset M$ peut être pris dans l'ouvert des points réguliers de $\Phi$. Ainsi pour $\xi^{\prime}$ suffisamment proche de $\xi, \mathcal{U} \cap \operatorname{Cr}\left(\left\|\Phi-\xi^{\prime}\right\|^{2}\right)=\Phi^{-1}\left(\xi^{\prime}\right)$ et $\partial \mathcal{U} \cap \operatorname{Cr}\left(\left\|\Phi-\xi^{\prime}\right\|^{2}\right)=\emptyset$, ce qui entraine $\mathrm{P}_{\xi^{\prime}}=\mathrm{P}_{\lambda_{\xi^{\prime}}}^{\mathcal{U}}$ dans $\mathcal{H}_{T, c}^{-\infty}(M)$. On a démontré le

Lemme 3.2 Soit $\xi$ une valeur régulière de $\Phi$. Pour $\xi^{\prime}$ suffisamment proche de $\xi$ on a $\mathrm{P}_{\xi^{\prime}}=\mathrm{P}_{\xi}$ dans $\mathcal{H}_{T, c}^{-\infty}(M)$.

En utilisant les lemmes 3.1 et 3.2 , on constate que $\mathrm{I}\left(M, \eta, \xi^{\prime}\right)=\mathrm{I}(M, \eta, \xi)$ si $\xi^{\prime}$ et $\xi$ appartiennent à la même composante connexe de valeurs régulières de $\Phi$.

Considérons maintenant le cas où $\xi \in \Delta$ est une valeur régulière de $\Phi_{\Delta}$. Ici $\Delta$ est un hyperplan séparant deux ouverts connexes de valeurs régulières de $\Phi$, et $\left(M_{\Delta}, T / T_{\Delta}, \Phi_{\Delta}\right)$ est la sous-variété hamiltonienne définie dans l'introduction. On choisit le voisinage ouvert $\mathcal{U}$ de $\Phi^{-1}(\xi)$ de telle manière que tout $m \in \mathcal{U}$ 
a un stabilisateur $\mathfrak{t}_{m}$ soit égal à $\mathfrak{t}_{\Delta}$, soit réduit à $\{0\}$. On a alors $\mathcal{U} \cap \operatorname{Cr}\left(\left\|\Phi-\xi^{\prime}\right\|^{2}\right)=\Phi^{-1}\left(\xi^{\prime}\right) \cup \Phi_{\Delta}^{-1}\left(\xi_{\Delta}^{\prime}\right)$ pour $\xi^{\prime}$ suffisamment proche de $\xi$, où $\xi_{\Delta}^{\prime}$ désigne le projeté orthogonal de $\xi^{\prime}$ sur $\Delta$ (voir la décomposition de $\operatorname{Cr}\left(\left\|\Phi-\xi^{\prime}\right\|^{2}\right)$ obtenue dans [6][Prop. 6.8]). Soient $\xi^{ \pm}$deux valeurs régulières de $\Phi$ qui sont séparées par $\Delta$, choisies suffisamment proches de $\xi$, et telles que leurs projetés orthogonaux sur $\Delta$ sont tous deux égaux à $\xi \in \Delta$. Comme $\mathcal{U} \cap \operatorname{Cr}\left(\left\|\Phi-\xi^{ \pm}\right\|^{2}\right)=\Phi^{-1}\left(\xi^{ \pm}\right) \cup \Phi_{\Delta}^{-1}(\xi)$, la propriété d'additivité vue à la section 2 donne $\mathrm{P}_{\lambda_{\xi^{ \pm}}}^{\mathcal{U}}=\mathrm{P}_{\xi^{ \pm}}+\mathrm{P}_{\lambda_{\xi^{ \pm}}}^{\mathcal{V}}$ où $\mathcal{V}$ est un ouvert adapté à $\lambda_{\xi^{ \pm}}$tel que $\mathcal{V} \cap \operatorname{Cr}\left(\left\|\Phi-\xi^{ \pm}\right\|^{2}\right)=\Phi_{\Delta}^{-1}(\xi)$. Comme les formes $\mathrm{P}_{\lambda_{\xi^{ \pm}}}^{\mathcal{U}}$ sont égales à $\mathrm{P}_{\xi}$ (voir (10)), on obtient $\mathrm{P}_{\xi^{+}}-\mathrm{P}_{\xi^{-}}=\mathrm{P}_{\lambda_{\xi^{-}}}^{\mathcal{V}}-\mathrm{P}_{\lambda_{\xi^{+}}}^{\mathcal{V}} \operatorname{dans} \mathcal{H}_{T, c}^{-\infty}(M)$.

Rappelons l'expression de $\mathrm{P}_{\lambda_{\xi^{ \pm}}}^{\mathcal{V}}$ obtenue en [6]. Sur la $T / T_{\Delta^{-}}$-variété hamiltonienne $\left(M_{\Delta}\right.$, $\left.\Phi_{\Delta}\right)$, l'élément $\xi \in \Delta$ permet de définir la classe $\mathrm{P}_{\xi}^{\Delta} \in \mathcal{H}_{T / T_{\Delta}, c}^{-\infty}\left(M_{\Delta}\right)$. D'après [6][Section 6.4], on a $\mathrm{P}_{\lambda_{\xi^{ \pm}}}^{\mathcal{V}}=$ $\left(i_{\Delta}\right)_{*}\left(\mathrm{P}_{\xi}^{\Delta} \operatorname{Eul}_{ \pm}^{-1}\left(N_{\Delta}\right)\right)$, où les inverses $\operatorname{Eul}_{ \pm}^{-1}\left(N_{\Delta}\right) \in \mathcal{C}^{-\infty}\left(\mathfrak{t}_{\Delta}, \mathcal{H}^{*}\left(M_{\Delta}\right)^{\text {bas }}\right)$ sont définis à la section $2:$ le produit de $\mathrm{P}_{\xi}^{\Delta} \in \mathcal{H}_{T / T_{\Delta}, c}^{-\infty}\left(M_{\Delta}\right)$ avec $\operatorname{Eul}_{ \pm}^{-1}\left(N_{\Delta}\right)$ détermine une classe $\mathrm{P}_{\xi}^{\Delta} \operatorname{Eul}_{ \pm}^{-1}\left(N_{\Delta}\right) \in \mathcal{H}_{T, c}^{-\infty}\left(M_{\Delta}\right)$. En utilisant la classe $\delta_{\Delta}^{o}=\operatorname{Eul}_{-}^{-1}\left(N_{\Delta}\right)-\operatorname{Eul}_{+}^{-1}\left(N_{\Delta}\right)$ définie en (6) on obtient la formule de saut suivante

Proposition $1 \quad \mathrm{P}_{\xi^{+}}-\mathrm{P}_{\xi^{-}}=\left(i_{\Delta}\right)_{*}\left(\mathrm{P}_{\xi}^{\Delta} \delta_{\Delta}^{o}\right) \quad$ dans $\quad \mathcal{H}_{T, c}^{-\infty}(M)$

Considérons le $T / T_{\Delta}$-fibré principal $\pi: \Phi_{\Delta}^{-1}(\xi) \rightarrow \mathcal{M}_{\xi}^{\Delta}$ et la restriction $\left.\delta_{\Delta}^{o}\right|_{\Phi_{\Delta}^{-1}(\xi)}$ de la classe $\delta_{\Delta}^{o} \in \mathcal{C}^{-\infty}\left(\mathfrak{t}_{\Delta}, \mathcal{H}^{*}\left(M_{\Delta}\right)^{\text {bas }}\right)$ : on a $\left.\delta_{\Delta}^{o}\right|_{\Phi_{\Delta}^{-1}(\xi)}=\pi^{*}\left(\delta_{\xi}^{o}\right)$ où $\delta_{\xi}^{o} \in \mathcal{C}^{-\infty}\left(\mathfrak{t}_{\Delta}, \mathcal{H}^{*}\left(\mathcal{M}_{\xi}^{\Delta}\right)\right)$ est supportée en 0 . Pour tout $\eta \in \mathcal{H}_{T}^{*}(M)$, la proposition 1 donne après intégration

$$
\int_{M \times \mathfrak{t}}\left(\mathrm{P}_{\xi^{+}}-\mathrm{P}_{\xi^{-}}\right)(X) \eta(X) \psi(X) d X=\frac{c_{\Delta}}{\left|S_{\xi}^{\Delta}\right|} \int_{\mathcal{M}_{\xi}^{\Delta} \times \mathfrak{t}_{\Delta}} \mathbf{K i r}_{\xi}^{\Delta}(\eta \psi)\left(X_{1}\right) \delta_{\xi}^{o}\left(X_{1}\right) d X_{1}
$$

avec $c_{\Delta}=(2 i \pi)^{d i m T-1} \operatorname{vol}\left(T / T_{\Delta}, d X_{2}\right)$ et $d X=d X_{1} d X_{2}$. Ici $\operatorname{Kir}_{\xi}^{\Delta}: \mathcal{H}_{T}^{\infty}(M) \rightarrow \mathcal{H}_{T_{\Delta}}^{\infty}\left(\mathcal{M}_{\xi}^{\Delta}\right)$ est le composé du morphisme de restriction $\mathcal{H}_{T}^{\infty}(M) \rightarrow \mathcal{H}_{T}^{\infty}\left(\Phi_{\Delta}^{-1}(\xi)\right)$ avec l'isomorphisme de Chern-Weil $\mathcal{H}_{T}^{\infty}\left(\Phi_{\Delta}^{-1}(\xi)\right) \stackrel{\sim}{\longrightarrow} \mathcal{H}_{T_{\Delta}}^{\infty}\left(\mathcal{M}_{\xi}^{\Delta}\right)$. L'élément $\delta_{\xi}^{o}$ détermine l'application $\operatorname{Res}_{\xi}^{o}: \mathcal{H}_{T_{\Delta}}^{*}\left(\mathcal{M}_{\xi}^{\Delta}\right) \rightarrow \mathcal{H}^{*}\left(\mathcal{M}_{\xi}^{\Delta}\right)$ en posant $\operatorname{Res}_{\xi}^{o}(\eta)=\frac{1}{2 i \pi} \int_{\mathfrak{t}_{\Delta}} \delta_{\xi}^{o}\left(X_{1}\right) \eta\left(X_{1}\right) \frac{d X_{1}}{\operatorname{vol}\left(T_{\Delta}, d X_{1}\right)}$. En prenant $\psi=1$ dans (11) on obtient $\mathrm{I}\left(M, \eta, \xi^{+}\right)-$ $\mathrm{I}\left(M, \eta, \xi^{-}\right)=\frac{1}{\left|S_{\xi}^{\Delta}\right|} \int_{\mathcal{M}_{\xi}^{\Delta}} \operatorname{Res}_{\xi}^{o} \circ \mathbf{K i r}_{\xi}^{\Delta}(\eta)$. Pour s'assurer que cette formule de saut correspond à (4) il suffit de vérifier que $\operatorname{Res}_{\xi}^{o} \circ \mathbf{K i r}_{\xi}^{\Delta}=\mathbf{K i r}_{M_{\Delta}, \xi} \circ \operatorname{Res}_{\Delta}^{o}$ où $\mathbf{K i r}_{M_{\Delta}, \xi}$ est le morphisme de Kirwan sur $\left(M_{\Delta}, T / T_{\Delta}, \Phi_{\Delta}\right)$ et $\operatorname{Res}_{\Delta}^{o}$ est l'application résidu définie par (7).

\section{Références}

[1] N. Berline, E. Getzler and M. Vergne, Heat kernels and Dirac operators, Grundlehren, vol. 298, Springer, Berlin, 1991.

[2] V. Guillemin and J. Kalkman, The Jeffrey-Kirwan localization theorem and residue operations in equivariant cohomology, J. Reine Angew. Math.,470, (1996), p. 123-142.

[3] V. Guillemin and S. Sternberg, Supersymmetry and equivariant de Rham theory. With an appendix containing two reprints by Henri Cartan. Mathematics Past and Present. Springer-Verlag, Berlin, 1999.

[4] F. Kirwan, Cohomology of quotients in symplectic and algebraic geometry, Princeton Univ. Press, Princeton, 1984.

[5] S. Kumar and M. Vergne, Equivariant cohomology with generalized coefficients, Astérisque, 215, 1993, p. 109-204.

[6] P-E. Paradan, Formules de localisation en cohomologie équivariante, Compositio Mathematica, 117, 1999, p. $243-293$.

[7] P-E. Paradan, The moment map and equivariant cohomology with generalized coefficients, Topology, 163, 2000, p. 401-444. 